\title{
Singularities of Positive Supersolutions in Elliptic PDEs
}

\author{
Louis Dupaigne and Augusto C. Ponce
}

Abstract. We present several extensions of the Brezis-Lions Lemma on removable singularities. We also give a positive answer to a question raised by $\mathrm{H}$. Brezis and M. Marcus about an "inverse" maximum principle for the Laplacian.

\section{Introduction and main results}

When can the set of singularities of a solution to a linear (or quasi-linear) elliptic equation be removed? To shed some light on this question, let us first recall a classical result in Potential Theory.

Here and throughout the rest of the paper, we assume that $\Omega \subset \mathbb{R}^{N}, N \geq 2$, is a bounded domain and $\Sigma \subset \Omega$ is a compact subset.

Let us assume that $\operatorname{cap}_{2}(\Sigma)=0$, where cap 2 denotes the standard $H^{1}$ capacity (see Section 2). Let $u \in H_{\text {loc }}^{1}(\Omega \backslash \Sigma)$ be a nonnegative function such that

$$
-\Delta u \geq 0 \quad \text { in } \mathcal{D}^{\prime}(\Omega \backslash \Sigma)
$$

Note that no information is given about $u$ on the set $\Sigma$. Nevertheless, it is well known that the function $u$ actually belongs to $L_{\text {loc }}^{1}(\Omega)$ and satisfies

$$
-\Delta u \geq 0 \quad \text { in } \mathcal{D}^{\prime}(\Omega)
$$

See, e.g., [11, Theorem 7.7]. Note that if $\operatorname{cap}_{2}(\Sigma)>0$, then (1.1) will no longer hold in general.

Our first theorem extends this classical result to the operator $-\Delta+c$, with $c \in \mathbb{R}$. It also generalizes a previous work of Brezis and Lions [4] (see also [7]), who considered the case where $\Sigma$ is a point:

Theorem 1 Assume that $\operatorname{cap}_{2}(\Sigma)=0$. Let $c \in \mathbb{R}$ and $f \in L_{\text {loc }}^{1}(\Omega)$. If $u \in$ $L_{\mathrm{loc}}^{1}(\Omega \backslash \Sigma), u \geq 0$ a.e. in $\Omega$, satisfies

$$
-\Delta u+c u \geq f \quad \text { in } \mathcal{D}^{\prime}(\Omega \backslash \Sigma),
$$

then $u \in L_{\mathrm{loc}}^{1}(\Omega)$ and

$$
-\Delta u+c u \geq f \quad \text { in } \mathcal{D}^{\prime}(\Omega)
$$

We would like to emphasize that we do not assume that $\Delta u \in L_{\text {loc }}^{1}(\Omega \backslash \Sigma)$.

Mathematics Subject Classification (2000). 35J15 (35B50).

Key words. Removable singularities, maximum principle. 
Remark 1 It follows from the proof of Theorem 1 that in fact $u \in W_{\text {loc }}^{1, p}(\Omega)$ for all $1 \leq p<\frac{N}{N-1}$; see also Corollary 6 . This regularity result is very standard and just follows from (1.3).

An interesting consequence of Theorem 1 is the following:

Corollary 2 Assume that $\operatorname{cap}_{2}(\Sigma)=0$. Let $c \in \mathbb{R}$ and $g: \mathbb{R}_{+} \rightarrow \mathbb{R}_{+}$be a continuous function. Let $u \in L_{\text {loc }}^{1}(\Omega \backslash \Sigma), u \geq 0$ a.e. in $\Omega$, be such that $g(u) \in$ $L_{\text {loc }}^{1}(\Omega \backslash \Sigma)$ and

$$
-\Delta u+c u \geq g(u) \quad \text { in } \mathcal{D}^{\prime}(\Omega \backslash \Sigma) .
$$

Then, $u, g(u) \in L_{\mathrm{loc}}^{1}(\Omega)$ and

$$
-\Delta u+c u \geq g(u) \quad \text { in } \mathcal{D}^{\prime}(\Omega)
$$

This corollary can be interpreted as a linear version of a very general result of Baras and Pierre [1] about removable singularities. Note that we do not impose any asymptotic behavior on $g(t)$ as $t \rightarrow \infty$.

We recall that any Radon measure $\mu$ in $\mathbb{R}^{N}$ can be decomposed as a sum $\mu=\mu_{\mathrm{a}}+\mu_{\mathrm{s}}$, where $\mu_{\mathrm{a}}$ and $\mu_{\mathrm{s}}$ are the absolutely continuous and the singular parts of $\mu$ with respect to the Lebesgue measure. There are several other possible decompositions of $\mu$ however. A less standard one is given by (see [3] and also [10])

$$
\mu=\mu_{\mathrm{d}}+\mu_{\mathrm{c}}
$$

where

$$
\begin{aligned}
& \mu_{\mathrm{d}}(A)=0 \quad \text { for any Borel set } A \subset \Omega \text { such that } \operatorname{cap}_{2}(A)=0, \\
& \left|\mu_{\mathrm{c}}\right|(\Omega \backslash F)=0 \quad \text { for some Borel set } F \subset \Omega \text { such that } \operatorname{cap}_{2}(F)=0 \text {. }
\end{aligned}
$$

In particular, the Radon measures $\mu_{\mathrm{d}}$ and $\mu_{\mathrm{c}}$ are singular with respect to each other.

Using the above notation, we have

Theorem 3 ("Inverse" maximum principle) Let $u \in L_{\mathrm{loc}}^{1}(\Omega)$ be such that $\Delta u$ is a Radon measure in $\Omega$. If $u \geq 0$ a.e. in $\Omega$, then

$$
(-\Delta u)_{\mathrm{c}} \geq 0 .
$$

We refer the reader to recent works of Brezis and Ponce [6], and also of Brezis, Marcus and Ponce [5], for some very nice applications of the "Inverse" maximum principle.

Theorem 1 can be extended to other second order linear elliptic operators. Here and in the rest of the paper, we use Einstein's summation convention. 
Theorem 4 Assume that $\operatorname{cap}_{2}(\Sigma)=0$. For $i, j \in\{1, \ldots, N\}$, let $a^{i j}, b^{i}, c \in$ $L^{\infty}(\Omega), f \in L^{1}(\Omega)$, and $g^{i} \in L^{2}(\Omega)$, where the coefficients $a^{i j}$ are locally Lipschitz continuous in $\Omega \backslash \Sigma$ and satisfy the uniform ellipticity condition

$$
a^{i j} \xi_{i} \xi_{j} \geq \lambda|\xi|^{2} \quad \forall \xi \in \mathbb{R}^{N}
$$

for some $\lambda>0$. If $u \in W_{\mathrm{loc}}^{1,1}(\Omega \backslash \Sigma), u \geq 0$ a.e. in $\Omega$, is such that

$$
-\partial_{j}\left(a^{i j} \partial_{i} u\right)+b^{i} \partial_{i} u+c u \geq f+\partial_{i} g^{i} \quad \text { in } \mathcal{D}^{\prime}(\Omega \backslash \Sigma),
$$

then $u \in W_{\text {loc }}^{1,1}(\Omega)$ and

$$
-\partial_{j}\left(a^{i j} \partial_{i} u\right)+b^{i} \partial_{i} u+c u \geq f+\partial_{i} g^{i} \quad \text { in } \mathcal{D}^{\prime}(\Omega) .
$$

Theorem 4 can be further generalized to the setting of quasi-linear elliptic equations as follows.

Let $A: \Omega \times \mathbb{R} \times \mathbb{R}^{N} \rightarrow \mathbb{R}^{N}$ and $B: \Omega \times \mathbb{R} \times \mathbb{R}^{N} \rightarrow \mathbb{R}$ be two Carathéodory functions. A weakly differentiable function $v$ in $\omega \subset \Omega$ is a supersolution of

$$
-\operatorname{div} A(x, v, \nabla v) \geq B(x, v, \nabla v) \quad \text { in } \mathcal{D}^{\prime}(\omega)
$$

if $A^{i}(x, v, \nabla v), B(x, v, \nabla v)$ are locally integrable in $\omega$ and

$$
\int_{\omega} A^{i}(x, v, \nabla v) \partial_{i} \varphi \geq \int_{\omega} B(x, v, \nabla v) \varphi \quad \forall \varphi \in C_{0}^{\infty}(\omega), \varphi \geq 0 \text { in } \omega .
$$

We shall assume in the sequel that $1<p \leq N$, and that for a.e. $x \in \Omega$ and every $r \geq 0, q \in \mathbb{R}^{N}$, we have

$$
\begin{aligned}
|A(x, r, q)| & \leq a_{0}|q|^{p-1}+a_{1} r^{p-1}+g(x), \\
-B(x, r, q) & \leq b_{0}|q|^{p-1}+b_{1} r^{p-1}+f(x), \\
A(x, r, q) \cdot q & \geq|q|^{p}-c_{1} r^{p}-c_{2},
\end{aligned}
$$

where $a_{i}, b_{i}, c_{i} \geq 0$ are constant, $f \in L^{1}(\Omega)$, and $g \in L^{p /(p-1)}(\Omega)$ are nonnegative functions.

Under these assumptions, we have the following:

Theorem 5 Suppose that $\operatorname{cap}_{p}(\Sigma)=0$. If $u \in W_{\text {loc }}^{1, p}(\Omega \backslash \Sigma), u \geq 0$ a.e. in $\Omega$, satisfies

$$
-\operatorname{div} A(x, u, \nabla u) \geq B(x, u, \nabla u) \quad \text { in } \mathcal{D}^{\prime}(\Omega \backslash \Sigma),
$$

then

$$
u^{p-1},|\nabla u|^{p-1} \in L_{\mathrm{loc}}^{1}(\Omega) .
$$

Furthermore, $A^{i}(x, u, \nabla u), B(x, u, \nabla u) \in L_{\mathrm{loc}}^{1}(\Omega)$ and $u$ satisfies

$$
-\operatorname{div} A(x, u, \nabla u) \geq B(x, u, \nabla u) \quad \text { in } \mathcal{D}^{\prime}(\Omega) .
$$


Here, $\operatorname{cap}_{p}(\Sigma)$ denotes the $W^{1, p}$-capacity of $\Sigma$ (see Definition 1 below).

Remark 2 The meaning of $\nabla u$ in $\Omega$ requires some clarification. In fact, since $u \in W_{\text {loc }}^{1, p}(\Omega \backslash \Sigma)$ and $|\Sigma|=0$, then $\nabla u$ is well defined a.e. in $\Omega$. We take this as the definition of $\nabla u$ in $\Omega$ even if $u$ is not (locally) weakly differentiable in the whole domain $\Omega$. By Corollary 6 below, if $p>2-\frac{1}{N}$, then $|\nabla u| \in L_{\text {loc }}^{1}(\Omega)$. In this case, we can conclude that $u \in W_{\text {loc }}^{1,1}(\Omega)$ and $\nabla u$ is the weak derivative of $u$ in $\Omega$ (see Lemma 3 below).

Remark 3 The fact that $A^{i}(x, u, \nabla u) \in L_{\text {loc }}^{1}(\Omega)$ is a direct consequence of (1.6) and (1.10). The corresponding property for $B(x, u, \nabla u)$ requires some additional argument.

The proof of Theorem 5 relies on a standard Moser iteration technique in the spirit of [15]. The same idea has been used by Serrin [14] to study removable singularities of solutions of

$$
-\operatorname{div} A(x, u, \nabla u)=B(x, u, \nabla u) \quad \text { in } \mathcal{D}^{\prime}(\Omega \backslash \Sigma) .
$$

Once (1.11) is established, then it is well known that the regularity result (1.10) can be improved. As we shall see in Section 5, we have

Corollary 6 Under the assumptions of Theorem 5, if $u \in W_{\mathrm{loc}}^{1, p}(\Omega \backslash \Sigma), u \geq 0$ a.e. in $\Omega$, satisfies (1.9), then

$$
\begin{gathered}
u^{p-1} \in L_{\mathrm{loc}}^{q}(\Omega) \quad \forall 1 \leq q<\frac{N}{N-p}, \\
|\nabla u|^{p-1} \in L_{\mathrm{loc}}^{r}(\Omega) \quad \forall 1 \leq r<\frac{N}{N-1} .
\end{gathered}
$$

We point out that Theorem 5 generalizes results of Bidaut-Véron [2] and also of Kilpeläinen [13] on removable singularities for the $p$-Laplace operator:

Corollary 7 Assume that $\operatorname{cap}_{p}(\Sigma)=0$. Let $c \in \mathbb{R}$ and $f \in L_{\text {loc }}^{1}(\Omega)$. If $u \in$ $W_{\mathrm{loc}}^{1, p}(\Omega \backslash \Sigma), u \geq 0$ a.e. in $\Omega$, satisfies

$$
-\Delta_{p} u+c u^{p-1} \geq f \quad \text { in } \mathcal{D}^{\prime}(\Omega \backslash \Sigma),
$$

then

$$
u^{p-1},|\nabla u|^{p-1} \in L_{\mathrm{loc}}^{1}(\Omega)
$$

and we have

$$
-\Delta_{p} u+c u^{p-1} \geq f \quad \text { in } \mathcal{D}^{\prime}(\Omega)
$$




\section{Some remarks about the $p$-capacity}

Given $1 \leq p<+\infty$, we first recall the definition of the $p$-capacity:

Definition 1 The $p$-capacity of a compact set $\Sigma \subset \Omega$ is defined as

$$
\operatorname{cap}_{p}(\Sigma)=\inf \left\{\int_{\Omega}|\nabla \varphi|^{p}: \varphi \in C_{0}^{\infty}(\Omega), \varphi \geq 1 \text { in some neighborhood of } \Sigma\right\} .
$$

It follows from Definition 1 that if $\operatorname{cap}_{p}(\Sigma)=0$, then $\operatorname{cap}_{q}(\Sigma)=0$ for every $1 \leq q<p$. We next point out that in this definition we could have restricted ourselves to a smaller class of functions $\varphi$. Namely, we have

$$
\operatorname{cap}_{p}(\Sigma)=\inf \left\{\begin{array}{l|l}
\int_{\Omega}|\nabla \varphi|^{p} & \begin{array}{l}
\varphi \in C_{0}^{\infty}(\Omega), 0 \leq \varphi \leq 1 \text { in } \Omega \\
\varphi=1 \text { in some neighborhood of } \Sigma
\end{array}
\end{array}\right\} .
$$

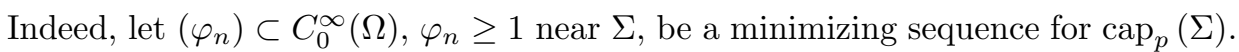
Define $v_{n}=\min \left(\varphi_{n}^{+}, 1\right)$ and observe that $v_{n}=1$ in a neighborhood of $\Sigma$. Denoting by $\left(\rho_{\varepsilon}\right)$ a sequence of standard mollifiers, it follows that for $\varepsilon=\varepsilon_{n}$ small enough, $w_{n}:=v_{n} * \rho_{\varepsilon_{n}}$ also satisfies $w_{n}=1$ in a neighborhood of $\Sigma$. Also $w_{n} \in C_{0}^{\infty}(\Omega)$, $w_{n} \geq 0$ in $\Omega$, and

$$
\int\left|\nabla w_{n}\right|^{p} \leq \int\left|\nabla \varphi_{n}\right|^{p} \rightarrow \operatorname{cap}_{p}(\Sigma)
$$

We also observe that if $\operatorname{cap}_{p}(\Sigma)=0$, then $|\Sigma|=0$. Indeed, it follows from Poincaré's inequality that for any nonnegative $\varphi \in C_{0}^{\infty}(\Omega)$ such that $\varphi=1$ on $\Sigma$, we have

$$
|\Sigma| \leq \int_{\Omega} \varphi^{p} \leq C \int_{\Omega}|\nabla \varphi|^{p}
$$

Taking the infimum with respect to $\varphi$, we conclude that $|\Sigma|=0$.

This result can be refined in more geometric terms (see [9] and also [8]):

Lemma 1 (i) $\mathcal{H}^{N-1}(\Sigma)=0$ if and only if $\operatorname{cap}_{1}(\Sigma)=0$;

(ii) if $1<p \leq N$ and $\mathcal{H}^{N-p}(\Sigma)<\infty$, then $\operatorname{cap}_{p}(\Sigma)=0$;

(iii) if $1<p \leq N$ and $\operatorname{cap}_{p}(\Sigma)=0$, then $\mathcal{H}^{s}(\Sigma)=0$ for every $s>N-p$;

(iv) if $p>N$ and $\operatorname{cap}_{p}(\Sigma)=0$, then $\Sigma=\phi$.

Note that (iv) is just a consequence of Morrey's inequality. In fact, if $p>N$ and $\left(\varphi_{n}\right) \subset C_{0}^{\infty}(\Omega)$ is such that $\int\left|\nabla \varphi_{n}\right|^{p} \rightarrow 0$, then $\left(\varphi_{n}\right)$ converges uniformly to 0 as $n \rightarrow \infty$. Since $\varphi_{n} \geq 1$ on $\Sigma$, we must have $\Sigma=\phi$. This shows in particular why, as mentioned earlier, we restrict ourselves to the case $p \leq N$.

As a corollary of Lemma 1 (ii), we have the following:

Corollary 8 Let $1<p \leq N$. If $\Sigma$ is contained in some manifold of codimension $k \geq p$, then $\operatorname{cap}_{p}(\Sigma)=0$. 
In this paper, we shall make use of the following two simple lemmas:

Lemma 2 Suppose $\operatorname{cap}_{p}(\Sigma)=0$. Given $\psi \in C_{0}^{\infty}(\Omega)$, there exists a sequence $\left(\psi_{n}\right) \subset C_{0}^{\infty}(\Omega \backslash \Sigma)$ such that

$$
\left|\psi_{n}\right| \leq|\psi| \quad \text { in } \Omega \quad \text { and } \quad \psi_{n} \rightarrow \psi \quad \text { in } W^{1, p}(\Omega) .
$$

If $\psi \geq 0$ in $\Omega$, then $\left(\psi_{n}\right)$ can be chosen so that each $\psi_{n}$ is nonnegative in $\Omega$.

Lemma 3 Suppose $\operatorname{cap}_{1}(\Sigma)=0$. If $u \in W^{1, p}(\Omega \backslash \Sigma)$, then $u \in W^{1, p}(\Omega)$.

Proof of Lemma 2. It suffices to take $\psi_{n}:=\left(1-\varphi_{n}\right) \psi$, where $\left(\varphi_{n}\right) \subset C_{0}^{\infty}(\Omega)$ is such that $0 \leq \varphi_{n} \leq 1$ in $\Omega, \varphi_{n}=1$ in some neighborhood of $\Sigma$, and $\int\left|\nabla \varphi_{n}\right|^{p} \rightarrow 0$ as $n \rightarrow \infty$.

Proof of Lemma 3. We split the proof into two steps:

Step 1. Assume in addition that $u$ is bounded. Then $u \in W^{1, p}(\Omega)$.

We first show that $u$ is weakly differentiable in $\Omega$. In fact, since $u$ is weakly differentiable in $\Omega \backslash \Sigma$, for each $i=1, \ldots, N$ we have

$$
\int_{\Omega} u \partial_{i} \varphi=-\int_{\Omega} \partial_{i} u \varphi \quad \forall \varphi \in C_{0}^{\infty}(\Omega \backslash \Sigma) .
$$

Given $\psi \in C_{0}^{\infty}(\Omega)$, it follows from the previous lemma that we can find a uniformly bounded sequence $\left(\psi_{n}\right)$ in $C_{0}^{\infty}(\Omega \backslash \Sigma)$ converging to $\psi$ in $W^{1,1}(\Omega)$. We now replace $\varphi$ by $\psi_{n}$ in the above identity. Passing to the limit as $n$ goes to $\infty$, we find that

$$
\int_{\Omega} u \partial_{i} \psi=-\int_{\Omega} \partial_{i} u \psi \quad \forall \psi \in C_{0}^{\infty}(\Omega) .
$$

In particular, $\partial_{i} u$ gives the weak derivative of $u$ in $\Omega$. Since

$$
\int_{\Omega}|\nabla u|^{p}=\int_{\Omega \backslash \Sigma}|\nabla u|^{p}<\infty,
$$

we conclude that $u \in W^{1, p}(\Omega)$.

Step 2. Proof of the lemma completed.

By working with the positive and negative parts of $u$, we may always assume that $u \geq 0$. For every $k>0$, let now $u_{k}=\min (u, k)$, so that $u_{k} \in W^{1, p}(\Omega \backslash \Sigma)$. It then follows from the previous step that $u_{k} \in W^{1, p}(\Omega)$ and

$$
\int_{\Omega} u_{k} \partial_{i} \psi=-\int_{\Omega} \partial_{i} u_{k} \psi=-\int_{[u \leq k]} \partial_{i} u \psi \quad \forall \psi \in C_{0}^{\infty}(\Omega) .
$$

Note that $\partial_{i} u \in L^{p}(\Omega \backslash \Sigma)=L^{p}(\Omega)$ for every $i=1, \ldots, N$. As $k \rightarrow \infty$, we conclude that $u$ is weakly differentiable in $\Omega$ and $u \in W^{1, p}(\Omega)$.

We now extend the definition of the $p$-capacity for any measurable subset of $\Omega$. For simplicity, we only consider the case $p=2$. 
Definition 2 Given an open set $\omega \subset \Omega$, we define

$$
\operatorname{cap}_{2}(\omega):=\sup \left\{\operatorname{cap}_{2}(K): K \text { is compact and } K \subset \omega\right\} .
$$

For any Borel measurable set $F \subset \Omega$, we let

$$
\operatorname{cap}_{2}(F):=\inf \left\{\operatorname{cap}_{2}(\omega): \omega \text { is open and } F \subset \omega \subset \Omega\right\} .
$$

One can easily see that Definition 2 agrees with Definition 1 when $F \subset \Omega$ is compact. We also observe that if $F_{1} \subset F_{2} \subset \Omega$, then $\operatorname{cap}_{2}\left(F_{1}\right) \leq \operatorname{cap}_{2}\left(F_{2}\right)$.

\section{Proof of Theorems 1 and 4}

The proof of Theorem 1 (and also of Theorem 4) is essentially contained in Section 5. However, it is enlightening to go through this special case before proving the more general result.

Below, we shall denote by $u_{k}$ the function $\min (u, k)$. Let us first state and prove the following fairly well known lemma:

Lemma 4 Let $u \in L^{1}(\omega), u \geq 0$ a.e. in $\omega$, be such that

$$
-\Delta u \geq h \quad \text { in } \mathcal{D}^{\prime}(\omega)
$$

where $h \in L^{1}(\omega)$. Then $u_{k} \in H_{\mathrm{loc}}^{1}(\omega)$ and

$$
-\Delta u_{k} \geq h \chi_{[u<k]} \quad \text { in } \mathcal{D}^{\prime}(\omega) .
$$

Proof. By taking convolution with a smooth mollifier on both sides of (3.1), we may assume that $u \in C^{\infty}$. It then follows from Kato's inequality (see [12]) that

$$
-\Delta u_{k} \geq h \chi_{[u<k]} \quad \text { in } \mathcal{D}^{\prime}(\omega) .
$$

We now multiply both sides of (3.3) by $\frac{\varphi^{2}}{u_{k}+1}$, where $\varphi \in C_{0}^{\infty}(\omega)$. Integrating by parts the resulting expression, we get

$$
\int_{\omega} \nabla u_{k} \cdot \nabla\left(\frac{\varphi^{2}}{u_{k}+1}\right) \geq-\int_{\omega}|h| \varphi^{2} .
$$

The left-hand side of (3.4) can be estimated by

$$
\begin{aligned}
\int_{\omega} \nabla u_{k} \cdot \nabla\left(\frac{\varphi^{2}}{u_{k}+1}\right) & =-\int_{\omega} \frac{\left|\nabla u_{k}\right|^{2}}{\left(u_{k}+1\right)^{2}} \varphi^{2}+2 \int_{\omega} \frac{\nabla u_{k} \cdot \nabla \varphi}{u_{k}+1} \varphi \\
& \leq-\frac{1}{2} \int_{\omega} \frac{\left|\nabla u_{k}\right|^{2}}{\left(u_{k}+1\right)^{2}} \varphi^{2}+2 \int_{\omega}|\nabla \varphi|^{2} .
\end{aligned}
$$


Since $u_{k}+1 \leq k+1$, we conclude from (3.4) and (3.5) that

$$
\frac{1}{2} \int_{\omega}\left|\nabla u_{k}\right|^{2} \varphi^{2} \leq(k+1)^{2} \int_{\omega}\left(|h| \varphi^{2}+2|\nabla \varphi|^{2}\right) .
$$

This was established assuming that $u \in C^{\infty}$. For a function $u \in L^{1}(\omega)$ satisfying (3.1), we can argue by density to conclude that $u_{k} \in H_{\text {loc }}^{1}(\omega)$ and that (3.2) holds.

Proof of Theorem 1. Applying the previous lemma to $\omega=\Omega \backslash \Sigma$, we see that $u_{k} \in H_{\mathrm{loc}}^{1}(\Omega \backslash \Sigma)$ for every $k>0$ and

$$
-\Delta u_{k}+c u \chi_{[u<k]} \geq f \chi_{[u<k]} \quad \text { in } \mathcal{D}^{\prime}(\Omega \backslash \Sigma) .
$$

Actually, we also established that

$$
\frac{1}{2} \int_{\Omega}\left|\nabla u_{k}\right|^{2} \varphi^{2} \leq(k+1)^{2} \int_{\Omega}\left(|\tilde{h}| \varphi^{2}+2|\nabla \varphi|^{2}\right) \quad \forall \varphi \in C_{0}^{\infty}(\Omega \backslash \Sigma),
$$

where $\tilde{h}=(f-c u) \chi_{[u<k]}$.

Let $\psi \in C_{0}^{\infty}(\Omega), \psi \geq 0$ in $\Omega$. Since $\operatorname{cap}_{2}(\Sigma)=0$, it follows from Lemma 2 that there exists a sequence $\left(\varphi_{n}\right) \subset C_{0}^{\infty}(\Omega \backslash \Sigma)$ such that $0 \leq \varphi_{n} \leq \psi$ and $\varphi_{n} \rightarrow \psi$ in $H^{1}(\Omega)$. We now replace $\varphi$ by $\varphi_{n}$ in (3.7). Passing to the limit as $n$ goes to $\infty$, we conclude that

$$
\frac{1}{2} \int_{\Omega}\left|\nabla u_{k}\right|^{2} \psi^{2} \leq(k+1)^{2} \int_{\Omega}\left(|\tilde{h}| \psi^{2}+2|\nabla \psi|^{2}\right)
$$

Take for instance $\psi=1$ in some neighborhood of $\Sigma$; Lemma 3 then implies that $u_{k} \in H_{\mathrm{loc}}^{1}(\Omega)$.

We now use $\varphi_{n}$ as a test function in (3.6):

$$
\int_{\Omega} \nabla u_{k} \cdot \nabla \varphi_{n}+c \int_{\Omega} u \chi_{[u<k]} \varphi_{n} \geq \int_{\Omega} f \chi_{[u<k]} \varphi_{n}
$$

Since $u_{k} \in H_{\mathrm{loc}}^{1}(\Omega)$, as $n \rightarrow \infty$ we find that

$$
\int_{\Omega} \nabla u_{k} \cdot \nabla \psi+c \int_{\Omega} u \chi_{[u<k]} \psi \geq \int_{\Omega} f \chi_{[u<k]} \psi \quad \forall \psi \in C_{0}^{\infty}(\Omega), \psi \geq 0 \text { in } \Omega .
$$

In other words,

$$
-\Delta u_{k}+c u \chi_{[u<k]} \geq f \chi_{[u<k]} \quad \text { in } \mathcal{D}^{\prime}(\Omega) .
$$

Assume for the moment that $u \in L_{\text {loc }}^{1}(\Omega)$. In this case, we are allowed to take $k \rightarrow \infty$ in (3.8), from which (1.3) follows.

Thus, in order to conclude the proof of Theorem 1, we only need to prove that $u \in L_{\text {loc }}^{1}(\Omega)$, which requires a Harnack type estimate. For this, we multiply both 
sides of (3.8) by $\frac{\varphi^{2}}{\left(u_{k}+1\right)^{2 / N}}$, where $\varphi \in C_{0}^{\infty}(\Omega)$. Proceeding exactly as in the previous lemma, we obtain

$$
\frac{1}{N} \int_{\Omega} \frac{\left|\nabla u_{k}\right|^{2}}{\left(u_{k}+1\right)^{\frac{N+2}{N}}} \varphi^{2} \leq|c| \int_{\Omega} u_{k}^{\frac{N-2}{N}} \varphi^{2}+\int_{\Omega}|f| \varphi^{2}+N \int_{\Omega}\left(u_{k}+1\right)^{\frac{N-2}{N}}|\nabla \varphi|^{2} .
$$

We claim that, by choosing $\varphi$ appropriately, this inequality implies that $u \in$ $L_{\text {loc }}^{1}(\Omega)$. Since the argument is essentially the same as in the more general setting (see Steps 2 and 3 in Section 5), we shall present here only a sketch of the proof.

We first take $\varphi=1$ in some small neighborhood $\omega$ of $\Sigma$. On the one hand, using Hölder's inequality, we have

$$
\int_{\Omega} u_{k}^{\frac{N-2}{N}} \varphi^{2} \leq|\omega|^{2 / N}\left(\int_{\omega} u_{k}\right)^{\frac{N-2}{N}}+C_{1}(\omega),
$$

where $C_{1}(\omega)$ denotes a constant independent of $k$. On the other hand, by the Sobolev inequality, there exists a constant $\alpha>0$ (independent of $\omega$ ) such that

$$
\alpha\left(\int_{\omega} u_{k}\right)^{\frac{N-2}{N}} \leq \int_{\Omega} \frac{\left|\nabla u_{k}\right|^{2}}{\left(u_{k}+1\right)^{\frac{N+2}{N}}} \varphi^{2}+C_{2}(\omega) .
$$

Combining (3.9)-(3.11), we get

$$
\left(1-\beta|\omega|^{N / 2}\right)\left(\int_{\omega} u_{k}\right)^{\frac{N-2}{N}} \leq C(\omega) .
$$

By choosing $|\omega|$ sufficiently small, it follows that $\left(u_{k}\right)$ is bounded in $L^{1}(\omega)$; thus, $u \in L_{\mathrm{loc}}^{1}(\Omega)$. This concludes the proof of Theorem 1 .

The proof of Theorem 4 follows along the same lines (although a little more technical) and we shall omit it.

\section{Proof of Corollary 2 and Theorem 3}

Proof of Corollary 2. Since $g(u) \geq 0$ a.e. in $\Omega$, the function $u$ satisfies

$$
-\Delta u+c u \geq 0 \quad \text { in } \mathcal{D}^{\prime}(\Omega \backslash \Sigma) .
$$

Applying Theorem 1 to $f=0$, we conclude that $u \in L_{\text {loc }}^{1}(\Omega)$ and

$$
-\Delta u+c u \geq 0 \text { in } \mathcal{D}^{\prime}(\Omega) .
$$

In particular, $\Delta u$ is a Radon measure in $\Omega$. By taking a smaller open set if necessary, we may assume that $\int_{\Omega}|\Delta u|<\infty$. 
Let $\left(\varphi_{n}\right) \subset C_{0}^{\infty}(\Omega \backslash \Sigma)$ be a nondecreasing sequence of test functions such that $0 \leq \varphi_{n} \leq 1$ in $\Omega$ and $\varphi_{n}(x) \rightarrow 1$ for every $x \in \Omega \backslash \Sigma$. It follows from (1.4) that

$$
\int_{\Omega} g(u) \varphi_{n} \leq-\int_{\Omega} \varphi_{n} \Delta u+c \int_{\Omega} \varphi_{n} u \leq \int_{\Omega}|\Delta u|+|c| \int_{\Omega}|u| .
$$

As $n \rightarrow \infty$, we conclude that

$$
\int_{\Omega} g(u) \leq \int_{\Omega}|\Delta u|+|c| \int_{\Omega}|u|<\infty
$$

(recall that $|\Sigma|=0$ ). Thus, $g(u) \in L_{\text {loc }}^{1}(\Omega)$ and clearly (1.5) holds.

Before establishing Theorem 3, we state the following variant of Lemma 4, which can be easily established via convolution:

Lemma 5 Let $u \in L^{1}(\omega), u \geq 0$ a.e. in $\omega$, be such that $\Delta u$ is a Radon measure in $\Omega$. Then $u_{k} \in H_{\mathrm{loc}}^{1}(\omega), \Delta u_{k}$ is a Radon measure in $\Omega$, and

$$
\Delta u_{k} \leq(\Delta u)^{+} \text {in } \mathcal{D}^{\prime}(\omega) .
$$

Proof of Theorem 3. It follows from the previous lemma applied to $\omega=\Omega$ that $u_{k} \in H_{\text {loc }}^{1}(\Omega), \forall k>0$. Let us simply denote $\Delta u$ by $\mu$ in $\Omega$. We fix a compact set $K \subset F$, where $F$ is a set of zero $H^{1}$-capacity such that $\left|\mu_{\mathrm{c}}\right|(\Omega \backslash F)=0$; in particular, $\operatorname{cap}_{2}(K)=0$. Applying Lemma 5 to $\omega=\Omega \backslash K$, we have

$$
\Delta u_{k} \leq \mu^{+} \quad \text { in } \mathcal{D}^{\prime}(\Omega \backslash K) .
$$

Given $\psi \in C_{0}^{\infty}(\Omega), \psi \geq 0$ in $\Omega$, let $\left(\varphi_{n}\right) \subset C_{0}^{\infty}(\Omega \backslash K)$ be such that $0 \leq \varphi_{n} \leq \psi$ in $\Omega$ and $\varphi_{n} \rightarrow \psi$ in $H^{1}(\Omega)$. Then

$$
\int_{\Omega} \nabla u_{k} \cdot \nabla \varphi_{n} \stackrel{n \rightarrow \infty}{\longrightarrow} \int_{\Omega} \nabla u_{k} \cdot \nabla \psi \quad \text { and } \quad \int_{\Omega} \varphi_{n} d \mu^{+} \leq \int_{\Omega \backslash K} \psi d \mu^{+} \quad \forall n \geq 1 .
$$

Combining (4.2) and (4.3), we conclude that

$$
-\int_{\Omega} \nabla u_{k} \cdot \nabla \psi \leq \int_{\Omega \backslash K} \psi d \mu^{+} \quad \forall \psi \in C_{0}^{\infty}(\Omega), \psi \geq 0 \text { in } \Omega ;
$$

in other words,

$$
\Delta u_{k} \leq \chi_{\Omega \backslash K} \mu^{+} \quad \text { in } \mathcal{D}^{\prime}(\Omega) .
$$

As $k \rightarrow \infty$, we get

$$
\mu=\Delta u \leq \chi_{\Omega \backslash K} \mu^{+} \quad \text { in } \mathcal{D}^{\prime}(\Omega)
$$

Thus,

$$
\mu_{\mathrm{c}}\left\lfloor_{K}=\mu\left\lfloor_{K} \leq 0 \quad \text { in } \Omega .\right.\right.
$$

Recall that $K \subset \Omega$ was an arbitrary compact subset of $F$. By the inner regularity of Radon measures, we finally conclude that

$$
\mu_{\mathrm{c}} \leq 0 \quad \text { in } \Omega \text {. }
$$




\section{$5 \quad$ Proof of Theorem 5}

By assumption, we know that $A^{i}(x, u, \nabla u), B(x, u, \nabla u) \in L_{\mathrm{loc}}^{1}(\Omega \backslash \Sigma)$, and

$$
\int_{\Omega} A^{i}(x, u, \nabla u) \partial_{i} \varphi \geq \int_{\Omega} B(x, u, \nabla u) \varphi \quad \forall \varphi \in C_{0}^{\infty}(\Omega \backslash \Sigma), \varphi \geq 0 \text { in } \Omega .
$$

Since $u \in W_{\text {loc }}^{1, p}(\Omega \backslash \Sigma)$ and $A$ satisfies (1.6), we actually have

$$
A^{i}(x, u, \nabla u) \in L_{\text {loc }}^{p /(p-1)}(\Omega \backslash \Sigma) .
$$

It follows from a density argument that

$$
\int_{\Omega} A^{i}(x, u, \nabla u) \partial_{i} v \geq \int_{\Omega} B(x, u, \nabla u) v
$$

for every $v \in W^{1, p}(\Omega) \cap L^{\infty}(\Omega)$ such that $v \geq 0$ a.e. in $\Omega$ and $\operatorname{supp} v \subset \Omega \backslash \Sigma$.

After replacing $u$ by $u+1$, we can assume that $u \geq 1$ a.e. in $\Omega$. Indeed, the function $v:=u+1$ satisfies

$$
-\operatorname{div} \tilde{A}(x, v, \nabla v) \geq \tilde{B}(x, v, \nabla v) \quad \text { in } \mathcal{D}^{\prime}(\Omega \backslash \Sigma),
$$

where $\tilde{A}(x, r, q)=A(x, r-1, q)$ and $\tilde{B}(x, r, q)=B(x, r-1, q)$. The functions $\tilde{A}$ and $\tilde{B}$ clearly verify assumptions (1.6)-(1.8).

We shall split the proof of Theorem 5 into three steps:

Step 1. For every $k \geq 1, u_{k} \in W_{\text {loc }}^{1, p}(\Omega)$. Moreover, given $0 \leq \sigma<p-1$, we have

$$
\int_{\Omega} \frac{\left|\nabla u_{k}\right|^{p}}{u_{k}^{p-\sigma}} \psi^{p} \leq C\left\{\int_{\Omega} u_{k}^{\sigma}\left(\psi^{p}+|\nabla \psi|^{p}\right)+\int_{\Omega} g^{p /(p-1)} \psi^{p}+\int_{\Omega} f \psi^{p}\right\}
$$

for all $\psi \in C_{0}^{\infty}(\Omega), \psi \geq 0$ in $\Omega$, where $C=C\left(p, \sigma, a_{i}, b_{i}, c_{i}\right)$.

Let $\varphi \in C_{0}^{\infty}(\Omega \backslash \Sigma)$ be such that $\varphi \geq 0$ in $\Omega$. We first apply (5.1) to

$$
v=w_{k} \varphi^{p}:=\left(\frac{1}{u_{k}^{p-\sigma-1}}-\frac{1}{k^{p-\sigma-1}}\right) \varphi^{p} \quad \text { in } \Omega .
$$

Note in particular that $v \geq 0$ in $\Omega$, and $v=0$ a.e. on the set $[u \geq k]$; hence, $\partial_{i} v=0$ a.e. on $[u \geq k]$. We have

$$
\begin{aligned}
\int_{\Omega} B(x, u, \nabla u) v & \leq \int_{\Omega} A^{i}(x, u, \nabla u) \partial_{i} v \\
& =\int_{[u<k]} A^{i}\left(x, u_{k}, \nabla u_{k}\right) \partial_{i}\left(w_{k} \varphi^{p}\right) \\
& =-(p-\sigma-1) \int_{\Omega} \frac{A^{i}\left(x, u_{k}, \nabla u_{k}\right) \partial_{i} u_{k}}{u_{k}^{p-\sigma}} \varphi^{p}+ \\
& \quad+p \int_{\Omega} A^{i}\left(x, u_{k}, \nabla u_{k}\right) w_{k} \partial_{i} \varphi \varphi^{p-1} .
\end{aligned}
$$


We now apply (1.8) to $r=u_{k}$ and $q=\nabla u_{k}$. Multiplying the resulting inequality by $\frac{\varphi^{p}}{u_{k}^{p-\sigma}}$ and integrating over $\Omega$, we get

$$
\int_{\Omega} \frac{\left|\nabla u_{k}\right|^{p}}{u_{k}^{p-\sigma}} \varphi^{p} \leq \int_{\Omega} \frac{A^{i}\left(x, u_{k}, \nabla u_{k}\right) \partial_{i} u_{k}}{u_{k}^{p-\sigma}} \varphi^{p}+\int_{\Omega} c_{1} u_{k}^{\sigma} \varphi^{p}+\int_{\Omega} c_{2} \frac{\varphi^{p}}{u_{k}^{p-\sigma}} .
$$

Combining (5.3) and (5.4) yields

$$
\int_{\Omega} \frac{\left|\nabla u_{k}\right|^{p}}{u_{k}^{p-\sigma}} \varphi^{p} \leq I+I I+\int_{\Omega} c_{1} u_{k}^{\sigma} \varphi^{p}+\int_{\Omega} c_{2} \varphi^{p}
$$

where

$$
\begin{aligned}
I & =-\frac{1}{p-\sigma-1} \int_{\Omega} B(x, u, \nabla u) v, \\
I I & =\frac{p}{p-\sigma-1} \int_{\Omega} A^{i}\left(x, u_{k}, \nabla u_{k}\right) w_{k} \partial_{i} \varphi \varphi^{p-1} .
\end{aligned}
$$

We first estimate (5.5). Since $\sigma<p-1$, we can apply (1.7) to get

$$
I \leq C \int_{\Omega}\left(b_{0}|\nabla u|^{p-1}+b_{1} u^{p-1}+f\right) v .
$$

Recall that $v=0$ a.e. on $[u \geq k]$ and $v \leq \frac{\varphi^{p}}{u_{k}^{p-\sigma-1}}$ a.e. in $\Omega$. We then have

$$
\begin{aligned}
I & \leq C \int_{[u<k]}\left(b_{0}|\nabla u|^{p-1}+b_{1} u^{p-1}+f\right) \frac{\varphi^{p}}{u_{k}^{p-\sigma-1}} \\
& \leq C\left\{\int_{\Omega} b_{0} \frac{\left|\nabla u_{k}\right|^{p-1}}{u_{k}^{p-\sigma-1}} \varphi^{p}+\int_{\Omega} b_{1} u_{k}^{\sigma} \varphi^{p}+\int_{\Omega} f \frac{\varphi^{p}}{u_{k}^{p-\sigma-1}}\right\} \\
& \leq C\left\{\int_{\Omega} b_{0} \frac{\left|\nabla u_{k}\right|^{p-1}}{u_{k}^{p-\sigma-1}} \varphi^{p}+\int_{\Omega} b_{1} u_{k}^{\sigma} \varphi^{p}+\int_{\Omega} f \varphi^{p}\right\} .
\end{aligned}
$$

We now estimate the first integral in the right-hand side of (5.7). We first write

$$
\int_{\Omega} b_{0} \frac{\left|\nabla u_{k}\right|^{p-1}}{u_{k}^{p-\sigma-1}} \varphi^{p}=\int_{\Omega} b_{0} \frac{\left|\nabla u_{k}\right|^{p-1}}{u_{k}^{(p-\sigma) \frac{p-1}{p}}} \varphi^{p-1} \cdot u_{k}^{\sigma / p} \varphi .
$$

For an arbitrary $\delta>0$, it follows from Young's inequality that

$$
\int_{\Omega} b_{0} \frac{\left|\nabla u_{k}\right|^{p-1}}{u_{k}^{p-\sigma-1}} \varphi^{p} \leq \delta \int_{\Omega} \frac{\left|\nabla u_{k}\right|^{p}}{u_{k}^{p-\sigma}} \varphi^{p}+C_{\delta} \int_{\Omega} u_{k}^{\sigma} \varphi^{p} .
$$


Inserting this into (5.7), we obtain

$$
I \leq \delta \int_{\Omega} \frac{\left|\nabla u_{k}\right|^{p}}{u_{k}^{p-\sigma}} \varphi^{p}+C_{\delta}\left\{\int_{\Omega} u_{k}^{\sigma} \varphi^{p}+\int_{\Omega} f \varphi^{p}\right\} .
$$

We now consider (5.6). Using (1.6) and arguing as above, we have

$$
\begin{aligned}
I I & =\frac{p}{p-\sigma-1} \int_{\Omega} A^{i}\left(x, u_{k}, \nabla u_{k}\right) w_{k} \partial_{i} \varphi \varphi^{p-1} \\
& \leq C \int_{[u<k]}\left(a_{0}|\nabla u|^{p-1}+a_{1} u^{p-1}+g\right) w_{k}|\nabla \varphi| \varphi^{p-1} \\
& \leq C\left\{\int_{\Omega} a_{0} \frac{\left|\nabla u_{k}\right|^{p-1}}{u_{k}^{p-\sigma-1}} \varphi^{p-1}|\nabla \varphi|+\int_{\Omega} a_{1} u_{k}^{\sigma} \varphi^{p-1}|\nabla \varphi|+\int_{\Omega} g \frac{\varphi^{p-1}}{u_{k}^{p-\sigma-1}}|\nabla \varphi|\right\} \\
& \leq C\left\{\int_{\Omega} a_{0} \frac{\left|\nabla u_{k}\right|^{p-1}}{u_{k}^{p-\sigma-1}} \varphi^{p-1}|\nabla \varphi|+\int_{\Omega} a_{1} u_{k}^{\sigma} \varphi^{p-1}|\nabla \varphi|+\int_{\Omega} g \varphi^{p-1}|\nabla \varphi|\right\} .
\end{aligned}
$$

On the other hand, given $\delta>0$, it follows from Young's inequality that

$$
\int_{\Omega} a_{0} \frac{\left|\nabla u_{k}\right|^{p-1}}{u_{k}^{p-\sigma-1}} \varphi^{p-1}|\nabla \varphi| \leq \delta \int_{\Omega} \frac{\left|\nabla u_{k}\right|^{p}}{u_{k}^{p-\sigma}} \varphi^{p}+C_{\delta} \int_{\Omega} u_{k}^{\sigma}|\nabla \varphi|^{p} .
$$

In addition,

$$
\begin{gathered}
\int_{\Omega} a_{1} u_{k}^{\sigma} \varphi^{p-1}|\nabla \varphi| \leq C\left\{\int_{\Omega} u_{k}^{\sigma} \varphi^{p}+\int_{\Omega} u_{k}^{\sigma}|\nabla \varphi|^{p}\right\}, \\
\int_{\Omega} g \varphi^{p-1}|\nabla \varphi| \leq \int_{\Omega}|\nabla \varphi|^{p}+\int_{\Omega} g^{p /(p-1)} \varphi^{p} .
\end{gathered}
$$

We now apply (5.9)-(5.12). Since $u_{k} \geq 1$ a.e. in $\Omega$, we get

$$
I I \leq \delta \int_{\Omega} \frac{\left|\nabla u_{k}\right|^{p}}{u_{k}^{p-\sigma}} \varphi^{p}+C_{\delta}\left\{\int_{\Omega} u_{k}^{\sigma}\left(\varphi^{p}+|\nabla \varphi|^{p}\right)+\int_{\Omega} g^{p /(p-1)} \varphi^{p}\right\} .
$$

Choosing $\delta>0$ sufficiently small, we conclude from (5.4), (5.8) and (5.13) that

$$
\int_{\Omega} \frac{\left|\nabla u_{k}\right|^{p}}{u_{k}^{p-\sigma}} \varphi^{p} \leq C\left\{\int_{\Omega} u_{k}^{\sigma}\left(\varphi^{p}+|\nabla \varphi|^{p}\right)+\int_{\Omega} g^{p /(p-1)} \varphi^{p}+\int_{\Omega} f \varphi^{p}\right\} .
$$

Let $\psi \in C_{0}^{\infty}(\Omega), \psi \geq 0$ in $\Omega$. Applying Lemma 2, we can find a sequence of nonnegative functions $\left(\psi_{n}\right)$ in $C_{0}^{\infty}(\Omega \backslash \Sigma)$ converging to $\psi$ in $W^{1, p}(\Omega)$ and a.e. in $\Omega$. Replacing $\varphi$ by $\psi_{n}$ in (5.14) and then letting $n \rightarrow \infty$ we find (5.2).

Let $\omega \subset \subset \Omega$ be some fixed open set containing $\Sigma$. We now take $\psi_{0} \in C_{0}^{\infty}(\Omega)$ so that $\psi_{0}=1$ on $\omega$ and $0 \leq \psi_{0} \leq 1$ in $\Omega$. Applying (5.2) with $\sigma=0$, we obtain

$$
\int_{\omega \backslash \Sigma}\left|\nabla u_{k}\right|^{p} \leq C k^{p}\left\{\int_{\Omega}\left(1+\left|\nabla \psi_{0}\right|^{p}\right)+\int_{\Omega} g^{p /(p-1)}+\int_{\Omega} f\right\} .
$$


In particular, $u_{k} \in W^{1, p}(\omega \backslash \Sigma)$. It follows from Lemma 3 that $u_{k} \in W^{1, p}(\omega)$. This concludes the first step of the proof.

Step 2. Given $0<\sigma<p-1$, we can find an open set $\omega \subset \subset \Omega$ containing $\Sigma$ so that

$$
\left\|u_{k}\right\|_{L^{\sigma \frac{N}{N-p}}(\omega)}^{\sigma}+\left\|\nabla u_{k}\right\|_{L^{\sigma} \frac{N}{N-1}(\omega)}^{\sigma} \leq C\left\{\int_{\Omega \backslash \bar{\omega}} u_{k}^{\sigma}+\int_{\Omega} g^{p /(p-1)}+\int_{\Omega} f\right\}
$$

for every $k \geq 1$, where $C=C\left(p, \sigma, \omega, \Omega, a_{i}, b_{i}, c_{i}\right)$.

Let $\omega \subset \subset \Omega$ be a neighborhood of $\Sigma$ with measure $|\omega|$ small enough to be chosen later on (recall that $|\Sigma|=0$, so that such $\omega$ actually exists). We then take $\psi_{0} \in C_{0}^{\infty}(\Omega)$, so that $\operatorname{supp} \psi_{0} \subset \Omega$ and $\psi_{0}=1$ on $\omega$. Since $u_{k} \in W_{\text {loc }}^{1, p}(\Omega)$ and $u_{k} \geq 1$ a.e. in $\Omega$, we have $u_{k}^{\sigma / p} \in W_{\text {loc }}^{1, p}(\Omega)$ and

$$
\nabla\left(u_{k}^{\sigma / p} \psi_{0}\right)=\frac{\sigma}{p} \frac{\nabla u_{k}}{u_{k}^{1-\sigma / p}} \psi_{0}+u_{k}^{\sigma / p} \nabla \psi_{0} \quad \text { in } \Omega .
$$

It follows from (5.2) that

$$
\int_{\Omega}\left|\nabla\left(u_{k}^{\sigma / p} \psi_{0}\right)\right|^{p} \leq C\left\{\int_{\Omega} u_{k}^{\sigma}\left(\psi_{0}^{p}+\left|\nabla \psi_{0}\right|^{p}\right)+\int_{\Omega} g^{p /(p-1)} \psi_{0}^{p}+\int_{\Omega} f \psi_{0}^{p}\right\} .
$$

Since $\nabla \psi_{0}=0$ on $\omega$, we get

$$
\begin{aligned}
\int_{\Omega}\left|\nabla\left(u_{k}^{\sigma / p} \psi_{0}\right)\right|^{p} & \leq C_{1} \int_{\omega} u_{k}^{\sigma}+C_{2}\left\{\left(1+\left\|\nabla \psi_{0}\right\|_{\infty}^{p}\right) \int_{\Omega \backslash \bar{\omega}} u_{k}^{\sigma}+\int_{\Omega} g^{p /(p-1)}+\int_{\Omega} f\right\} \\
& \leq C_{1} \int_{\omega} u_{k}^{\sigma}+C_{2} K,
\end{aligned}
$$

where $K$ denotes the term in brackets and $C_{1}, C_{2}$ are positive constants independent of $k$; note also that $C_{1}$ does not depend on $\omega$.

Applying the Sobolev inequality, we find that

$$
\begin{aligned}
& \left(\int_{\omega} u_{k}^{\sigma \frac{N}{N-p}}\right)^{\frac{N-p}{N}} \leq\left(\int_{\Omega} u_{k}^{\sigma \frac{N}{N-p}} \psi_{0}^{\frac{N p}{N-p}}\right)^{\frac{N-p}{N}} \\
& \leq \tilde{C}_{1} \int_{\omega} u_{k}^{\sigma}+\tilde{C}_{2} K \quad \text { if } 1<p<N, \\
& \left(\int_{\omega} u_{k}^{\sigma q}\right)^{\frac{1}{q}} \leq \tilde{C}_{1} \int_{\omega} u_{k}^{\sigma}+\tilde{C}_{2} K \quad \forall q \in[1, \infty) \quad \text { if } p=N,
\end{aligned}
$$

where $\tilde{C}_{1}$ is independent of $\omega$.

We shall assume in the sequel that $1<p<N$, since the case $p=N$ can be dealt with in a similar way. From Hölder's inequality, we know that

$$
\int_{\omega} u_{k}^{\sigma} \leq|\omega|^{p / N}\left(\int_{\omega} u_{k}^{\sigma \frac{N}{N-p}}\right)^{\frac{N-p}{N}} .
$$


Inserting (5.18) into (5.16), we find that

$$
\left(1-|\omega|^{p / N} \tilde{C}_{1}\right) \int_{\omega} u_{k}^{\sigma} \leq|\omega|^{p / N} \tilde{C}_{2} K
$$

We now choose $\omega$ so that $|\omega|^{p / N} \tilde{C}_{1}<1 / 2$. Thus, we have

$$
\frac{1}{2} \int_{\omega} u_{k}^{\sigma} \leq|\omega|^{p / N} \tilde{C}_{2} K
$$

We finally conclude from (5.16) and the above that

$$
\left(\int_{\omega} u_{k}^{\sigma \frac{N}{N-p}}\right)^{\frac{N-p}{N}} \leq\left(2|\omega|^{p / N} \tilde{C}_{1}+1\right) \tilde{C}_{2} K \leq 2 \tilde{C}_{2} K .
$$

This gives the estimate for the first term in the left-hand side of (5.15). We now estimate the second one.

Applying Hölder's inequality, we have

$$
\begin{aligned}
\int_{\omega}\left|\nabla u_{k}\right|^{\sigma \frac{N}{N-1}} & =\int_{\omega} \frac{\left|\nabla u_{k}\right|^{\sigma \frac{N}{N-1}}}{u_{k}^{(p-\sigma) \frac{\sigma}{p} \frac{N}{N-1}}} \cdot u_{k}^{(p-\sigma) \frac{\sigma}{p} \frac{N}{N-1}} \\
& \leq\left(\int_{\omega} \frac{\left|\nabla u_{k}\right|^{p}}{u_{k}^{p-\sigma}}\right)^{\frac{\sigma}{p} \frac{N}{N-1}}\left(\int_{\omega} u_{k}^{\sigma \frac{N}{N-p /(p-\sigma)}}\right)^{1-\frac{\sigma}{p} \frac{N}{N-1}} .
\end{aligned}
$$

By (5.2) and (5.19), the first integral is bounded by $C K$. Note that $\frac{N}{N-p /(p-\sigma)}<$ $\frac{N}{N-p}$ for $\sigma<p-1$; thus, by Hölder's inequality, the second integral can be estimated by $C K^{\frac{N}{N-p /(p-\sigma)}}$. Therefore,

$$
\int_{\omega}\left|\nabla u_{k}\right|^{\sigma \frac{N}{N-1}} \leq(C K)^{\frac{\sigma}{p} \frac{N}{N-1}}\left(C K^{\frac{N}{N-p /(p-\sigma)}}\right)^{1-\frac{\sigma}{p} \frac{N}{N-1}}=C K^{\frac{N}{N-1}} .
$$

This concludes the proof of Step 2.

Step 3. Proof of (1.10).

Since $u \in W_{\text {loc }}^{1, p}(\Omega \backslash \Sigma)$, it suffices to show that $u^{p-1},|\nabla u|^{p-1}$ are integrable in some small neighborhood of $\Sigma$. Given $0<\sigma<p-1$, it follows from the previous step that (5.15) holds for some small open set $\omega$ containing $\Sigma$. In particular,

$$
\int_{\omega} u_{k}^{\sigma \frac{N}{N-p}} \leq C\left\{\int_{\Omega \backslash \bar{\omega}} u^{\sigma}+\int_{\Omega} g^{p /(p-1)}+\int_{\Omega} f\right\}^{\frac{N}{N-p}} \quad \forall k \geq 1 .
$$

(Shrinking the domain $\Omega$ if necessary, we can always assume that $\int_{\Omega \backslash \bar{\omega}} u^{\sigma}<\infty$ ). 
By making the special choice $\sigma=(p-1) \frac{N-p}{N}$ in (5.21), we immediately see that $u^{p-1} \in L^{1}(\omega)$. Note also that according to (5.15), we have

$$
\int_{\omega}\left|\nabla u_{k}\right|^{\sigma \frac{N}{N-1}} \leq C\left\{\int_{\Omega \backslash \bar{\omega}} u^{\sigma}+\int_{\Omega} g^{p /(p-1)}+\int_{\Omega} f\right\}^{\frac{N}{N-1}} \quad \forall k \geq 1 .
$$

Take in particular $\sigma=(p-1) \frac{N-1}{N}$. Since $|\Sigma|=0$ and $u \in W_{\text {loc }}^{1, p}(\Omega \backslash \Sigma)$, we have $\nabla u_{k}=\chi_{[u<k]} \nabla u$ a.e. in $\Omega$. Applying the Monotone Convergence Theorem to (5.22), we conclude that (1.10) holds.

The argument above actually shows that (1.12) and (1.13) hold; moreover, we have

$$
\|u\|_{L^{\sigma} \frac{N}{N-p}(\omega)}^{\sigma}+\|\nabla u\|_{L^{\sigma} \frac{N}{N-1}(\omega)}^{\sigma} \leq C\left\{\int_{\Omega \backslash \bar{\omega}} u^{\sigma}+\int_{\Omega} g^{p /(p-1)}+\int_{\Omega} f\right\},
$$

where $C=C\left(p, \sigma, \omega, \Omega, a_{i}, b_{i}, c_{i}\right)$ and $0<\sigma<p-1$.

Step 4. $A^{i}(x, u, \nabla u), B(x, u, \nabla u) \in L_{\mathrm{loc}}^{1}(\Omega)$ and

$$
-\operatorname{div} A(x, u, \nabla u) \geq B(x, u, \nabla u) \quad \text { in } \mathcal{D}^{\prime}(\Omega) .
$$

In view of (1.10) and the structure estimate $(1.6), A^{i}(x, u, \nabla u) \in L_{\text {loc }}^{1}(\Omega)$. Given $k>0$, let $F_{k} \in C^{\infty}(\mathbb{R})$ be a non-increasing function such that $F_{k}(t)=1$ if $t \leq k / 2, F_{k}(t)=0$ if $t \geq k$ and $\left|F_{k}^{\prime}\right| \leq 4 / k$ in $\mathbb{R}$. Since $F_{k}$ is non-increasing, we have in particular that $0 \leq F_{k} \leq 1$ in $\mathbb{R}$.

Note that $F_{k} \circ u=F_{k} \circ u_{k}$. As a consequence of the first step, we thus have

$$
F_{k} \circ u \in W_{\mathrm{loc}}^{1, p}(\Omega) \quad \text { and } \quad \nabla\left(F_{k} \circ u\right)=F_{k}^{\prime}(u) v \nabla u \quad \text { a.e. in } \Omega .
$$

Given $\varphi \in C_{0}^{\infty}(\Omega \backslash \Sigma), \varphi \geq 0$ in $\Omega$, it follows from (5.1) applied to the function $v=F_{k}(u) \varphi$ that

$$
\begin{aligned}
\int_{\Omega} B(x, u & , \nabla u) F_{k}(u) \varphi \leq \\
& \leq \int_{\Omega} A^{i}(x, u, \nabla u) \partial_{i} u F_{k}^{\prime}(u) \varphi+\int_{\Omega} A^{i}(x, u, \nabla u) \partial_{i} \varphi F_{k}(u) \\
& =\int_{\Omega} A^{i}\left(x, u_{k}, \nabla u_{k}\right) \partial_{i} u_{k} F_{k}^{\prime}(u) \varphi+\int_{\Omega} A^{i}\left(x, u_{k}, \nabla u_{k}\right) \partial_{i} \varphi F_{k}(u),
\end{aligned}
$$

where we have used the fact that $F_{k}(t)=F_{k}^{\prime}(t)=0$ for all $t \geq k$.

Given $\psi \in C_{0}^{\infty}(\Omega), \psi \geq 0$ in $\Omega$, let $\left(\psi_{n}\right)$ be a sequence of nonnegative functions in $C_{0}^{\infty}(\Omega \backslash \Sigma)$ converging to $\psi$ with respect to the $W^{1, p}$-norm and also a.e. in $\Omega$.

We first observe that in view of (1.7) and (1.10), we have

$$
B(x, u, \nabla u) \geq-b_{0}|\nabla u|^{p-1}-b_{1} u^{p-1}-f \in L_{\mathrm{loc}}^{1}(\Omega) .
$$


It follows from Fatou's Lemma that

$$
\int_{\Omega} B(x, u, \nabla u) F_{k}(u) \psi \leq \liminf _{n \rightarrow \infty} \int_{\Omega} B(x, u, \nabla u) F_{k}(u) \psi_{n} .
$$

We now apply $(5.23)$ with $\varphi$ replaced by $\psi_{n}$. Since $A^{i}\left(x, u_{k}, \nabla u_{k}\right) \in L_{\text {loc }}^{p /(p-1)}(\Omega)$, we can take $n \rightarrow \infty$ in the resulting inequality to get

$$
\begin{aligned}
\int_{\Omega} B(x, u, \nabla u) F_{k}(u) \psi \leq \int_{\Omega} A^{i}\left(x, u_{k}, \nabla\right. & \left.u_{k}\right) \partial_{i} u_{k} F_{k}^{\prime}(u) \psi+ \\
& +\int_{\Omega} A^{i}\left(x, u_{k}, \nabla u_{k}\right) \partial_{i} \psi F_{k}(u),
\end{aligned}
$$

for every $\psi \in C_{0}^{\infty}(\Omega)$ such that $\psi \geq 0$ in $\Omega$.

We now let $k \rightarrow \infty$ in the inequality above. By Fatou's Lemma,

$$
\int_{\Omega} B(x, u, \nabla u) \psi \leq \liminf _{k \rightarrow \infty} \int_{\Omega} B(x, u, \nabla u) F_{k}(u) \psi
$$

Next, since

$$
\begin{aligned}
\left|A\left(x, u_{k}, \nabla u_{k}\right)\right| & \leq a_{0}\left|\nabla u_{k}\right|^{p-1}+a_{1} u_{k}^{p-1}+g \\
& \leq a_{0}|\nabla u|^{p-1}+a_{1} u^{p-1}+g \in L_{\mathrm{loc}}^{1}(\Omega),
\end{aligned}
$$

it follows from the Dominated Convergence Theorem that

$$
\lim _{k \rightarrow \infty} \int_{\Omega} A^{i}\left(x, u_{k}, \nabla u_{k}\right) \partial_{i} \psi F_{k}(u)=\int_{\Omega} A^{i}(x, u, \nabla u) \partial_{i} \psi .
$$

Finally, recall that $-4 / k \leq F_{k}^{\prime} \leq 0$ in $\mathbb{R}$. Using (1.8), we have

$$
\begin{aligned}
\int_{\Omega} A^{i}\left(x, u_{k}, \nabla u_{k}\right) \partial_{i} u_{k} F_{k}^{\prime}(u) \psi & \leq \int_{\Omega}\left(\left|\nabla u_{k}\right|^{p}-c_{1} u_{k}^{p}-c_{2}\right) F_{k}^{\prime}(u) \psi \\
& \leq \frac{4}{k} \int_{\left[\frac{k}{2}<u<k\right]}\left(\left|c_{1}\right| u_{k}^{p}+\left|c_{2}\right|\right) \psi
\end{aligned}
$$

Since $u_{k}^{p} / k \leq u_{k}^{p-1} \leq u^{p-1}$, we get

$$
\int_{\Omega} A^{i}\left(x, u_{k}, \nabla u_{k}\right) \partial_{i} u_{k} F_{k}^{\prime}(u) \psi \leq 4 \int_{\left[\frac{k}{2}<u<k\right]}\left(\left|c_{1}\right| u^{p-1}+\frac{\left|c_{2}\right|}{k}\right) \psi \rightarrow 0
$$

as $k \rightarrow \infty$. It then follows from (5.24)-(5.27) that

$$
\int_{\Omega} B(x, u, \nabla u) \psi \leq \int_{\Omega} A^{i}(x, u, \nabla u) \partial_{i} \psi \quad \forall \psi \in C_{0}^{\infty}(\Omega), \psi \geq 0 \text { in } \Omega .
$$

In particular, since $B(x, u, \nabla u)$ is bounded from below by an $L_{\text {loc }}^{1}$-function in $\Omega$, we must have $B(x, u, \nabla u) \in L_{\mathrm{loc}}^{1}(\Omega)$. 


\section{Acknowledgments}

We are indebted to H. Brezis and M. Marcus for conjecturing Theorem 3 in a personal communication. We would also like to thank H. Brezis for his encouragement. The second author (A.C.P.) was partially supported by CAPES, Brazil, under grant no. BEX1187/99-6.

\section{References}

[1] P. Baras and M. Pierre. Singularités éliminables pour des équations semilinéaires. Ann. Inst. Fourier (Grenoble) 34 (1984), 185-206.

[2] M.-F. Bidaut-Véron. Local and global behavior of solutions of quasilinear equations of Emden-Fowler type. Arch. Rational Mech. Anal. 107 (1989), 293-324

[3] R.M. Blumenthal and R.K. Getoor. Markov processes and potential theory. Pure and Applied Mathematics, Vol. 29. Academic Press, New York, 1968.

[4] H. Brezis and P.-L. Lions. A note on isolated singularities for linear elliptic equations. Mathematical analysis and applications, Part A. Adv. in Math. Suppl. Stud., Vol. 7. Academic Press, New York, 1981, pp. 263-266.

[5] H. Brezis, M. Marcus, and A.C. Ponce. Nonlinear elliptic equations with measures revisited. In preparation.

[6] H. Brezis and A.C. Ponce. Kato's inequality when $\Delta u$ is a measure. C. $R$. Math. Acad. Sci. Paris, Ser. I 338 (2004), 599-604.

[7] J. Dávila and A.C. Ponce. Variants of Kato's inequality and removable singularities. J. Anal. Math. 91 (2003), 143-178.

[8] L.C. Evans and R.F. Gariepy. Measure theory and fine properties of functions. Studies in Advanced Mathematics. CRC Press, Boca Raton, FL, 1992.

[9] H. Federer and W.P. Ziemer. The Lebesgue set of a function whose distribution derivatives are $p$-th power summable. Indiana Univ. Math. J. 22 (1972/73), 139-158.

[10] M. Fukushima, K. Sato, and S. Taniguchi. On the closable parts of preDirichlet forms and the fine supports of underlying measures. Osaka J. Math. 28 (1991), 517-535.

[11] L.L. Helms. Introduction to Potential Theory. Pure and Applied Mathematics, Vol. XXII. Wiley-Interscience, New York, 1969. 
[12] T. Kato. Schrödinger operators with singular potentials. Proceedings of the International Symposium on Partial Differential Equations and the Geometry of Normed Linear Spaces (Jerusalem, 1972). Israel J. Math. 13 (1972), 135148 (1973).

[13] T. Kilpeläinen. Potential theory for supersolutions of degenerate elliptic equations. Indiana Univ. Math. J. 38 (1989), 253-275.

[14] J. Serrin. Local behavior of solutions of quasi-linear equations. Acta Math. 111 (1964), 247-302.

[15] N.S. Trudinger. On Harnack type inequalities and their application to quasilinear elliptic equations. Comm. Pure Appl. Math. 20 (1967), 721-747.

L. Dupaigne

Laboratoire Amiénois de Mathématique Fondamentale et Appliquée

Université Picardie Jules Verne

Faculté de Mathématiques et d'Informatique

33, rue Saint-Leu

80039 Amiens Cedex 1, France

e-mail: louis.dupaigne@u-picardie.fr

A.C. Ponce

Laboratoire Jacques-Louis Lions

Université Pierre et Marie Curie

Boîte courier 187

75252 Paris Cedex 05, France

e-mail: ponce@ann.jussieu.fr

Rutgers University

Department of Mathematics, Hill Center, Busch Campus

110 Frelinghuysen Rd.

Piscataway, NJ 08854, USA

e-mail: augponce@math.rutgers.edu 